\title{
ON LOGNORMAL RANDOM VARIABLES: I-THE CHARACTERISTIC FUNCTION
}

\author{
ROY B. LEIPNIK
}

(Received 21 July 1989; revised 28 February 1990)

\begin{abstract}
The characteristic function of a lognormal random variable is calculated in closed form as a rapidly convergent series of Hermite functions in a logarithmic variable. The series coefficients are Nielsen numbers, defined recursively in terms of Riemann zeta functions. Divergence problems are avoided by deriving a functional differential equation, solving the equation by a de Bruijn integral transform, expanding the resulting reciprocal Gamma function kernel in a series, and then invoking a convergent termwise integration. Applications of the results and methods to the distribution of a sum of independent, not necessarily identical lognormal variables are discussed. The result is that a sum of lognormals is distributed as a sum of products of lognormal distributions. The case of two lognormal variables is outlined in some detail.
\end{abstract}

\section{Introduction}

Lognormal distributions are widely and increasingly used to fit data from radar, physical and biological lifetimes, incomes, stock market prices, geography and geology. They also appear in Brownell's (1955) method of regularizing quantum mechanical calculations.

The paper is divided into four sections. Section 1 is devoted to basic formulas and to the derivation of a functional differential equation for the characteristic function. Section 2 takes up the application of the de Bruijn integral transform to solving the equation, and to proving that there is a unique solution of the de Bruijn type which was the correct Fourier transform, involving a reciprocal Gamma function. The non-uniqueness problem for lognormal moments is illustrated.

\footnotetext{
'Mathematics Department, University of California at Santa Barbara, CA 93106 USA.

(C) Copyright Australian Mathematical Society 1991, Serial-fee code 0334-2700/91
} 
Section 3 carries out through the tedious detail of expanding the reciprocal Gamma function in Taylor series, term by term integration, proof of convergence, and coefficient evaluation. Section 4 indicates how the functional differential equation for the characteristic function can be used to derive a functional equation for the embedding function, a natural and useful extension of the sum distribution, and to obtain a partially determined form of the sum distribution as a mixture of lognormal distributions.

In many of these fields, the distribution of a sum of independent lognormal variables (perhaps with different parameters) is of scientific interest. An often convenient approach to sum problems is via the characteristic function (normalised Fourier transform) of the distribution. The relative simplicity of the lognormal distribution and of its moments and Mellin transform is deceptive-the successful path to calculating the characteristic function is rather roundabout, in order to avoid divergent series. The final result is quite complicated, and therefore of primary interest to the specialist.

However, the methods are helpful in approaching the yet more difficult problem of the sum distributions. A complete treatment of the latter will be found in a second paper on lognormal distributions, denoted throughout by II.

In the middle 1970's, the author was studying the effect of the atmosphere on radar signals; in radar noise theory, molecular particles are modeled as having a gaussian velocity distribution, and dust particles of a given type as having a lognormal distribution. The sum of a gaussian variable and one or more lognormal variables is therefore important. The usual approach to sums of independent random variables is via characteristic functions (c.f.'s), i.e., Fourier transforms. Surprisingly, the c.f. of the lognormal and also the distribution of the sum of 2 lognormals were not in the literature (as of 1975).

In 1980, the author obtained the lognormal c.f. by formal manipulation using the method of de Bruijn, explained later. Professor Kotlarski, a Polish probabilist then visiting USCB, remarked that the same problem had long been considered as a "Matterhorn" by East-Zone probabilists, and was unsolved in 1978. Unfortunately, the many double-limit interchanges were difficult to justify by conventional methods, and the validity of the results was questioned. Nevertheless, the result was disclosed to some radar theorists, along with an application to the sum of a gaussian and one lognormal, but not then published. However, one spin-off of the technique was published as a counterexample [10] and reprinted by Crow and Shimano [4], (Dr. Crow is a former colleague who has used the lognormal in telecommunications studies). While on sabbatical at Adelaide University in June 1989, a revisiting of the problem by this writer yielded the fairly technical proofs included below.

Another bibliographical point: a manuscript on lognormal distributions by 
the British statistician Holgate was disclosed to me by Dr. Crow in August 1989. Holgate did not touch the exact result, but his approximations are interesting and useful; they overlap slightly the elliptic function counterexample results published in the perhaps obscure USSR reference (see [10]). Since the book of Crow and Shimano appeared very recently, Dr. Holgate quite probably had not noticed this item in it. Holgate uses methods more familiar than the undetermined kernel method of de Bruijn, favored by Dutch statistical physicists (such as Van Hove) in asymptotic studies. It is flexible enough to fit many characteristic function problems, as explained later in this paper. In particular, normal distributions transformed by inverses of solutions of linear differential equations are susceptible to the de Bruijn method. The author has seen several technical reports in the radar field in which the present results are applied. Understandably, they are not very readable by the non-radar audience.

\section{A functional differential equation for the lognormal characteristic function}

The lognormal probability density is defined for $0<x<\infty$ as

$$
p\left(\mu, \sigma^{2}, x\right)=\left(2 \pi \sigma^{2}\right)^{-1 / 2} x^{-1} \exp \left\{-(\log x-\mu)^{2} / 2 \sigma^{2}\right\},
$$

where $0<\sigma$, and $\mu$ is real.

The Mellin transform of $p$ is given by

$$
\begin{aligned}
M\left(\mu, \sigma^{2}, s\right) & =E\left[X^{s}\right] \\
& =\left(2 \pi \sigma^{2}\right)^{-1 / 2} \int_{0}^{\infty} x^{s-1} \exp \left\{-(\log x-\mu)^{2} / 2 \sigma^{2}\right\} d x \\
& =\left(2 \pi \sigma^{2}\right)^{-1 / 2} \int_{-\infty}^{\infty} \exp \left\{s y-(y-\mu)^{2} / 2 \sigma^{2}\right\} d y \\
& =\exp \left(s \mu+s^{2} \sigma^{2} / 2\right)
\end{aligned}
$$

where $X$ is a lognormal random variable whose density is $p\left(\mu, \sigma^{2}, x\right)$.

For further reference it is convenient to separate $M$ into $M^{+}$and $M^{-}$. Here

$$
\begin{aligned}
M^{+}\left(\mu, \sigma^{2}, s\right) & =\left(2 \pi \sigma^{2}\right)^{-1 / 2} \int_{1}^{\infty} x^{s-1} \exp \left[-(\log x-\mu)^{2} / 2 \sigma^{2}\right] d x \\
& =(1 / 2) \exp \left(s \mu+s^{2} \sigma^{2} / 2\right) \cdot \operatorname{erfc}\left(-(\sigma s+\mu / \sigma) / 2^{1 / 2}\right)
\end{aligned}
$$

by Magnus et al ([12], p. 350). As usual, we write ([12], p. 349)

$$
\operatorname{erf} c(u)=1-\operatorname{erf}(u)=1-\frac{2}{\sqrt{\pi}} \int_{0}^{u} e^{-t^{2}} d t .
$$


Similarly,

$$
\begin{aligned}
M^{-}\left(\mu, \sigma^{2}, s\right) & =\left(2 \pi \sigma^{2}\right)^{-1 / 2} \int_{0}^{1} x^{s-1} \exp \left\{-(\log x-\mu)^{2} / 2 \sigma^{2}\right\} d x \\
& =(1 / 2) \exp \left(s \mu+s^{2} \sigma^{2} / 2\right) \operatorname{er} f c\left((\sigma s+\mu / \sigma) / 2^{1 / 2}\right),
\end{aligned}
$$

so that $M=M^{+}+M^{-}$follows from the fact $\operatorname{erf}$ is an odd function.

The integer moments of $p$ are given by

$$
\mu_{n}=M\left(\mu, \sigma^{2}, n\right)=\exp \left(n \mu+n^{2} \sigma^{2} / 2\right) .
$$

The obvious approach to calculation of the characteristic function

$$
\phi\left(\mu, \sigma^{2}, t\right)=\left(2 \pi \sigma^{2}\right)^{-1 / 2} \int_{0}^{\infty} x^{-1} \exp \left\{-(\log x-\mu)^{2} / 2 \sigma^{2}\right\} \exp (i t x) d x
$$

is to write $\exp ($ it $x)=\sum_{n=0}^{\infty}(i t x)^{n} / n$ ! and obtain by termwise integration

$$
\phi\left(\mu, \sigma^{2}, t\right)=\sum_{n=0}^{\infty}(i t)^{n} \mu_{n} / n !
$$

Unfortunately, the ratio

$$
\begin{aligned}
\left|\frac{(i t)^{n+1} \mu_{n+1}}{(n+1) !} /\left(\frac{(i t)^{n} \mu_{n}}{n !}\right)\right| & =\frac{|t|}{n+1} \exp \left\{(n+1-n) \mu+\frac{\left\{(n+1)^{2}-n^{2}\right\} \sigma^{2}}{2}\right\} \\
& =\frac{|t|}{n+1} \exp \left\{\mu+\left(n+\frac{1}{2}\right) \sigma^{2}\right\}
\end{aligned}
$$

tends to infinity for each $t \neq 0, \sigma \neq 0$ as $n \rightarrow \infty$.

This divergence is a great nuisance, as it necessitates an entirely different approach. Another consequence of the rapid increase of $\mu_{n}$ is that

$$
\left|\mu_{n}\right|^{1 / n}=\exp \left(\mu+n \sigma^{2} / 2\right) \quad \text { and so } \sum_{n}\left|\mu_{n}\right|^{-1 /(2 n)}<\infty \text {. }
$$

Thus the Carleman [3] sufficient condition for unique determination of the distribution from its moments is violated, as is the condition of Krein [9]. That this uniqueness fails, and indeed fails spectacularly, we shall show explicitly in Section 2.

Since we shall later be interested in non-identical lognormal variables, we shall occasionally write (from (1) and (7))

$$
p_{j}(x)=p\left(\mu_{j}, \sigma_{j}^{2}, x\right), \quad \phi_{j}(t)=\phi\left(\mu_{j}, \sigma_{j}^{2}, t\right) \quad \text { for } j=1,2, \ldots
$$

Under suitable conditions (Titchmarsh [16]) the integral (7) can be inverted, yielding

$$
p\left(\mu, \sigma^{2}, x\right)=\frac{1}{2 \pi} \int_{-\infty}^{\infty} \exp (-i x t) \phi\left(\mu, \sigma^{2}, t\right) d t .
$$


Since $\phi\left(\mu, \sigma^{2},-t\right)=\phi^{*}\left(\mu, \sigma^{2}, t\right)$,

$$
p(x)=\frac{1}{\pi} \int_{0}^{\infty}\{\cos (x t) \Re \phi(t)+\sin (x t) \mathfrak{I} \phi(t)\} d t
$$

can be calculated from $\phi(t)$ for $t>0$ only.

Some relevant facts about $p(x)$ are now listed.

(i) $p(x)$ is continuous and infinitely differentiable on $(0, \infty)$.

(ii) $\lim _{x \rightarrow 0^{+}} p^{(j)}(x)=0$ for every $j$, so that $p(x)$ has infinite order contact with the $x$-axis at $x=0$ (by convention, $p(x)=0$ for $x<0)$.

(iii) $p(x)$ is unimodal with its maximum at $x=\exp \left(\mu-\sigma^{2}\right)$.

(iv) The function

$$
\left\{x^{r}(p(x))\right\}^{q}=\text { const } x^{q(r-1)} \exp \left\{-q(\log x-\mu)^{2} / 2 \sigma^{2}\right\}
$$

is a lognormal density (up to a scale factor in $\log x$ ) and so $x^{r} p(x)$ is in $L^{q}(0, \infty)$ for each $q>0$ and each real $r$.

From Titchmarsh [16] (pp. 11-12, p. 72, pp. 75-76, pp. 83-86, pp. 107108, Theorem 79) the following three statements are implied by the previous four statements:

$$
\begin{gathered}
\lim _{t \rightarrow \pm \infty} \phi(t)=0, \\
p(x)=\lim _{A \rightarrow \infty} \frac{1}{2 \pi} \int_{-A}^{A} \exp (-i t x) \phi(t) d t \\
=\lim _{A \rightarrow \infty} \frac{1}{\pi} \int_{0}^{A}\{\cos (t x) \Re \phi(t)+\sin (t x) \mathfrak{I} \phi(t)\} d t
\end{gathered}
$$

for each $x>0$, and there is a finite bound $K_{q}$ for the $L^{q}$ norm of $\phi$, defined by

and (Plancherel's theorem)

$$
\int_{-\infty}^{\infty}|\phi(t)|^{q} d t \leq K_{q} \int_{0}^{\infty} x^{q-2}(p(x))^{q} d x
$$

$$
\int_{-\infty}^{\infty}|\phi(t)|^{2} d t=2 \pi \int_{0}^{\infty}|p(x)|^{2} d x
$$

Several identities for $\phi$ are helpful. (The operations needed are justified by the preceding paragraph.) We first note that

$$
\begin{aligned}
\phi\left(\mu, \sigma^{2}, t\right) & \left.=\left(2 \pi \sigma^{2}\right)^{-1 / 2} \int_{-\infty}^{\infty} \exp \left\{i t e^{y}-(y-\mu)^{2} / 2 \sigma^{2}\right)\right\} d y \\
& =\left(2 \pi \sigma^{2}\right)^{-1 / 2} \int_{-\infty}^{\infty} \exp \left\{i t e^{(y+\mu)}-y^{2} / 2 \sigma^{2}\right\} d y \\
& =\phi\left(0, \sigma^{2}, t e^{\mu}\right)
\end{aligned}
$$


so that

$$
\phi\left(\mu_{1}+\nu_{1}, \sigma^{2}, t\right)=\phi\left(\mu_{1}, \sigma^{2}, t e^{\nu_{1}}\right) .
$$

By completing the square, we obtain

$$
k y-(y-\mu)^{2} / 2 \sigma^{2}=-\left(y-\mu_{k}^{*}\right)^{2} / 2 \sigma^{2}+\left\{\mu_{k}^{* 2}-\mu^{2}\right\} / 2 \sigma^{2}
$$

where $\mu_{k}^{*}=\mu+\sigma^{2} k$.

Hence, from (14) (differentiating under the integral sign) and (15), we obtain a functional equation of the type called a functional differential equation, namely

$$
\frac{d}{d t} \phi\left(\mu, \sigma^{2}, t\right)=i\left(2 \pi \sigma^{2}\right)^{-1 / 2} \int_{-\infty}^{\infty} \exp \left\{i t \exp y+y-(y-\mu)^{2} / 2 \sigma^{2}\right\} d t
$$

Using (a) with $k=1$,

$$
\begin{aligned}
\frac{d}{d t} \phi\left(\mu, \sigma^{2}, t\right)= & \left.i\left(2 \pi \sigma^{2}\right)^{-1 / 2} \exp \left\{\mu_{1}^{* 2}-\mu^{2}\right) / 2 \sigma^{2}\right\} \\
& \times \int_{-\infty}^{\infty} \exp \left\{i t e^{y}-\left(y-\mu_{1}^{*}\right)^{2} / 2 \sigma^{2}\right\} d y \\
& =i K \phi\left(\mu_{1}^{*}, \sigma^{2}, t\right) \\
& =i K \phi\left(\mu, \sigma^{2}, L t\right)
\end{aligned}
$$

where

$$
K=\exp \left\{\mu+\left(\sigma^{2} / 2\right)\right\} \quad \text { and } \quad L=\exp \left(\sigma^{2}\right) .
$$

The equation (16) is the key to the whole development below. Its solutions are not unique per se, but together with the fact that the Fourier transform of $\phi$ is known, uniqueness can be verified, within the subclass possessing the given transform.

\section{Solutions of the functional differential equation}

Two rather different solution methods can be found in the literature. Each solves the equation up to an unknown function which must be determined by initial, boundary, (or Fourier transform) conditions.

The method due to Pinney [14], produces a long and quite unusable expression, based on Titchmarsh's finite Laplace transform. A second method, due to de Bruijn [2], is based on a Gamma function, or Barnes-Mellin transform, and is much more convenient in the present case. A third and novel method, based on Jacobi series, is mentioned because of its relation to nonuniqueness. 
The first step in de Bruijn's method is to obtain a difference-differential equation by a change of variable. From (14), it is sufficient to consider the case $\mu=0$. Let

$$
\chi(t)=\phi\left(e^{t}\right)
$$

so that

$$
\chi\left(t+\sigma^{2}\right)=\phi\left(\exp \left(t+\sigma^{2}\right)\right)=\phi\left(e^{t} \cdot e^{\sigma^{2}}\right) .
$$

Hence by (16) and (17), we have

$$
\begin{aligned}
\chi^{\prime}(t) & =\phi^{\prime}(\exp t) \cdot \exp t \\
& =i \exp \left(t+\sigma^{2} / 2\right) \phi\left(e^{t} e^{\sigma^{2}}\right) \\
& =i \exp \left(t+\sigma^{2} / 2\right) \chi\left(t+\sigma^{2}\right)
\end{aligned}
$$

as the desired (forward) difference-differential equation (with variable coefficient). The development below is intended to motivate the somewhat surprising expression (28) for the solutions of (20).

De Bruijn's functional is

$$
\chi_{S}(t)=Q \int_{k-i \infty}^{k+i \infty} \exp \left\{a z^{2}+b z+c z t\right\} S(z) \Gamma(z) d z
$$

where $S(z)$ is an arbitrary function, $\Gamma(z)$ is the gamma function, $Q$ is a constant, and $S(z) \Gamma(z)$ is analytic in a strip containing the lines $(k-$ $i \infty, k+i \infty)$ and $(k+1-i \infty, k+1+i \infty)$. The contour lies in this strip. The resulting family of solutions of (20) is large enough to include the $\chi_{S}$ whose corresponding $\phi_{S}$ is the particular $\phi$ required.

Clearly

$$
\chi_{S}\left(t+\sigma^{2}\right)=Q \int_{k-i \infty}^{k+1 \infty} \exp \left\{a z^{2}+\left(b+c \sigma^{2}\right) z+c z t\right\} S(z) \Gamma(z) d z
$$

and

$$
\chi_{S}^{\prime}(t)=Q c \int_{k-i \infty}^{k+i \infty} \exp \left\{a z^{2}+b z+c z t\right\} S(z) z \Gamma(z) d z .
$$

Shifting the line of integration within the strip and making use of $\Gamma(z+1)=z \Gamma(z)$ we find

$$
\chi_{S}^{\prime}(t)=Q c \int_{k+1-i \infty}^{k+1+i \infty} \exp \left\{a(z-1)^{2}+b(z-1)+c(z-1) t\right\} S(z-1) \Gamma(z) d z .
$$

If now two special conditions are imposed:

$$
\begin{gathered}
S(z-1)=-S(z), \text { and } \\
i \exp \left\{\sigma^{2} / 2+a z^{2}+\left(b+c \sigma^{2}\right) z+(c z+1) t\right\} \\
=-c \exp \left\{a(z-1)^{2}+b(z-1)+c(z-1) t\right\}
\end{gathered}
$$


for all $z, t$, then (20) is satisfied, since the integrand is by assumption analytic in the strip between the two vertical lines of integration.

Thus $S(z)$ is anti-periodic with anti-period 1 , and $a, b, c$ satisfy

$$
\begin{aligned}
i \exp \left(\sigma^{2} / 2\right) & =-c \exp (a-b) \\
c \sigma^{2} & =-2 a \\
1 & =-c
\end{aligned}
$$

whose solutions are

$$
a=\sigma^{2} / 2, \quad b=-(2 \nu+1 / 2) \pi i, \quad \text { and } \quad c=-1
$$

where $\nu$ is an integer.

Thus

$$
\chi_{S}(t)=Q \int_{k-\imath \infty}^{k+l \infty} \exp \left\{\sigma^{2} z^{2} / 2-z t-(2 \nu+1 / 2) \pi i z\right\} S(z) \Gamma(z) d z
$$

is a solution for all $t$. This method of solution by an ansatz is used widely in special function theory.

From (18), the corresponding function $\phi_{S}(t)$ is defined for $t>0$ by

$$
\phi_{S}(t)=Q \int_{k-i \infty}^{k+i \infty} \exp \left\{\sigma^{2} z^{2} / 2-z \log t-(2 \nu+1 / 2) \pi i z\right\} S(z) \Gamma(z) d z
$$

and for $t<0$ by $\phi_{S}(t)=\phi_{S}^{*}(-t)$.

We now wish to find choices of $S(z)$ and $\nu$ which yield the correct characteristic function $\phi(t)$ of the lognormal distribution, if possible. To this end, let

$$
f_{S}^{+}(x)=\frac{1}{2 \pi} \int_{0}^{\infty} \exp (-i x t) \phi_{S}(t) d t .
$$

Thus

$$
\begin{aligned}
f_{S}^{+}(x)=(2 \pi)^{-1} Q \int_{0}^{\infty} \int_{k-i \infty}^{k+i \infty} \exp (-i x t) & \\
& \times \exp \left\{\sigma^{2} z^{2} / 2-z \log t-(2 \nu+1 / 2) \pi i z\right\} S(z) \Gamma(z) d z d t \\
=(2 \pi)^{-1} Q \int_{k-i \infty}^{k+i \infty} \exp \left\{\sigma^{2} z^{2} / 2-(2 \nu+1 / 2) \pi i z\right\} S(z) \Gamma(z) d z & \\
& \times \int_{0}^{\infty} t^{-z} \exp (-i x t) d t
\end{aligned}
$$

if the function $S(z)$ is such that the integrals can be interchanged. We later verify that for the $S(z)$ of interest, the interchange is valid.

Now by Erdelyi ([5], p. 118)

$$
\int_{0}^{\infty} t^{-z} \exp (-i x t) d t=x^{z-1} \exp \{\pi i(z-1) / 2\} \Gamma(1-z) \text { for } 0<\mathfrak{R}<<1 \text {. }
$$


Hereafter, we take $0<k<1$ in the vertical integration line, to permit use of (32). Thus substituting (32) into (31)

$$
\begin{aligned}
f_{S}^{+}(x)=(2 \pi)^{-1} Q \int_{k-i \infty}^{k+i \infty} & \exp \left\{\sigma^{2} z^{2} / 2-(2 \nu+1 / 2) \pi i z\right\} \frac{\pi}{\sin (\pi z)} \\
& \times x^{z-1} \exp \{\pi i(z-1) / 2\} S(z) d z \\
= & -i(Q / 2) \int_{k-i \infty}^{k+i \infty} x^{z-1} \exp \left\{\sigma^{2} z^{2} / 2-2 \nu \pi i z\right\} S(z) \frac{d z}{\sin \pi z}
\end{aligned}
$$

since $\Gamma(z) \Gamma(1-z)=\pi / \sin \pi z$ and $\exp (-i \pi / 2)=-i$.

Also define

$$
\begin{aligned}
f_{S}^{-}(x) & =(2 \pi)^{-1} \int_{-\infty}^{0} \exp (i x t) \phi_{S}(t) d t \\
& =(2 \pi)^{-1} \int_{0}^{\infty} \exp (i x t) \phi_{S}^{*}(t) d t \\
& =\left(f_{S}^{+}(x)\right)^{*} .
\end{aligned}
$$

Hence the inverse Fourier transform of $\phi_{S}(t)$ is

$$
\begin{aligned}
f_{S}(x) & =(2 \pi)^{-1} \int_{-\infty}^{\infty} \exp (-i x t) \phi_{S}(t) d t \\
& =f_{S}^{+}(x)+f_{S}^{-}(x) \\
& =2 \mathfrak{R} f_{S}^{+}(x) .
\end{aligned}
$$

Note that (33) can be interpreted as a complex Laplace transform. We now express $p\left(0, \sigma^{2}, x\right)$ as a complex transform. Compute

$$
\begin{aligned}
J & =\int_{k-i \infty}^{k+i \infty} \exp \left(a z^{2}+\xi z\right) d z \\
& =i \int_{-\infty}^{\infty} \exp \left\{a(i x)^{2}+\xi(i x)\right\} d x \\
& =i(\pi / a)^{1 / 2} \exp \left(-\xi^{2} / 4 a\right) .
\end{aligned}
$$

The shift from complex to real integrals in (36) is permitted by integrating around the rectangle whose vertices are $k+i N, k-i N,-i N, i N$, where $N$ is large. The integrand is analytic in the rectangle. Moreover, the contributions on the horizontal segments are bounded by

$$
\exp \left(-A N^{2}\right) \int_{0}^{K} \exp \left(a \mu^{2}+\xi \mu\right) d \mu
$$

which approaches 0 as $N \rightarrow \infty$. Hence as $N \rightarrow \infty$, the integral

$$
J_{N}=\int_{k-i N}^{k+i N} \exp \left\{a z^{2}+b z\right\} d z
$$


and the integral

$$
\int_{-i N}^{i N} \exp \left\{a z^{2}+b z\right\} d z=i \int_{-i N}^{N} \exp \left\{-a u^{2}-i b u\right\} d u
$$

tend to the same limit, namely $i \int_{-\infty}^{\infty} \exp \left\{-a u^{2}-i b u\right\} d z$. Thus for $x>0$, $a=\sigma^{2} / 2, b=\log x,(36)$ implies

$\int_{k-1 \infty}^{k+i \infty} \exp \left(\sigma^{2} z^{2} / 2\right) x^{z-1} d z=i x^{-1}\left(2 \pi / \sigma^{2}\right)^{1 / 2} \exp \left\{-(\log x)^{2} / 2 \sigma^{2}\right\}=2 \pi i p(x)$

which is consistent with the Mellin transform formula (2).

Now if

$$
S(z)=\sum_{p=0}^{\infty} a_{p} \sin (2 p+1) \pi z
$$

then $S(z-1)=-S(z)$. Taking $Q$ real, $a_{p}=\delta_{p, 0}$, and $\nu=0$, we find that $f_{S}^{+}(x)$ is real on comparing (33) and (37). More precisely, the function

$$
\left\{[\exp (-2 \nu \pi i z) / \sin (\pi z)\} \cdot \sum_{p=0}^{\infty} a_{p} \sin (2 p+1) \pi z-1\right\}
$$

must be orthogonal to $\exp \left(\sigma^{2} z^{2} / 2\right) x^{z-1}$ for all $x$, which implies $\nu=0$ and $a_{p}=\delta_{p, 0}$. Hence

$$
f_{S}(x)=2 \pi Q p(x)=p(x) \text { for all } x>0 \text { if } Q=(2 \pi)^{-1} .
$$

The uniqueness of the Fourier (complex Laplace) transforms also implies that $(2 \pi)^{-1} \sin (\pi z)$ is the unique $Q S(z)$ which fulfills the requirement $f_{S}(x)=$ $p(x)$ for all $x>0$. Thus from (29), for all $t>0,0<k<1$,

$$
\phi\left(0, \sigma^{2}, t\right)=(2 \pi)^{-1} \int_{k-i \infty}^{k+i \infty} \exp \left\{\sigma^{2} z^{2} / 2-z(\log (t)+\pi i / 2)\right\}(\sin (\pi z) \Gamma(z)) d z,
$$

or alternatively

$$
\phi\left(0, \sigma^{2}, t\right)=\frac{1}{2} \cdot \int_{k-i \infty}^{k+i \infty} \exp \left\{\sigma^{2} z^{2} / 2-z(\log (t)+\pi i / 2)\right\} d z / \Gamma(1-z)
$$

Another approach to the functional differential equation is now explored. Let

$$
\psi_{a}(t)=\sum_{n=-\infty}^{\infty} a^{-n} L^{-n^{2} / 2} \exp \left(i a L^{n} t\right) / w(a)
$$


where $w(a)=\sum_{n=-\infty}^{\infty} a^{-n} L^{-n^{2} / 2}, \quad a>0$ and $L>1$. This Jacobi series, and all of its derivatives, converges absolutely and uniformly in $t$. Note that

$$
\psi_{a}^{\prime}(t)=\sum_{n=-\infty}^{\infty} a^{-n}\left(i a L^{n}\right) L^{-n^{2} / 2} \exp \left(i a L^{n} t\right) / w(a)
$$

$$
\begin{aligned}
i K \psi_{a}(L t) & =i K \sum_{n=-\infty}^{\infty} a^{-n} L^{-n^{2} / 2} \exp \left(i a L^{n+1} t\right) / w(a) \\
& =i K \sum_{n} a^{-(n-1)} L^{-(n-1)^{2} / 2} \exp \left(i a L^{n} t\right) / w(a) \\
& =i a K L^{-1 / 2} \sum_{n} a^{-n} L^{n-n^{2} / 2} \exp \left(i a L^{n} t\right) / w(a) .
\end{aligned}
$$

But $K L^{-1 / 2}=\exp \left(\sigma^{2} / 2\right)\left(\exp \sigma^{2}\right)^{-1 / 2}=1$ so that

$$
\psi_{a}^{\prime}(t)=i K \psi_{a}(L t)
$$

as desired. Clearly the $\psi_{a}(t)$ are strongly dependent on $a$. However, $\psi_{a}$ is the characteristic function of a discrete distribution $p_{a}(x)$ which places weights $\lambda(a, n)=a^{-n} L^{-n^{2} / 2} / w(a)$ on the points $x_{n}(a)=a L^{n}$ for $n=$ $0, \pm 1, \pm 2, \ldots$, where $L=\exp \bar{\sigma}^{2}$, and thus cannot prossibly be the characteristic function of the continuous density $p(x)$. Note that $p_{a}$ is unimodal, except for ties.

The moment sequence of every $p_{a}$ is exactly the same as that of $p$, for compute

$$
\begin{aligned}
\left.L^{-q^{2} / 2} i^{-q}\left(\frac{\partial}{\partial t}\right)^{q} \psi_{z}(t)\right|_{t=0} & =L^{-q^{2} / 2} i^{-q} \sum_{n=-\infty}^{\infty}\left(i a L^{n}\right)^{q} a^{-n} L^{-n^{2} / 2} / w(a) \\
& =\sum_{n=-\infty}^{\infty} a^{-(n-q)} L^{-(n-q)^{2} / 2} / w(a)=1
\end{aligned}
$$

so that $\mu_{q}\left(p_{a}\right) / \mu_{q}(p)=1$ for each $q$ and $a>0$.

One hopes that for some mixing distribution $d h(a)$, we have

$$
p(x)=\int_{0}^{\infty} p_{a}(x) d h(a) \text { and } \int_{0}^{\infty} d h(a)=1
$$

and so too

$$
\phi(t)=\int_{0}^{\infty} \phi_{a}(t) d h(a)
$$

Unfortunately, the necessary $d h(a)$ is somewhat complicated and so sheds 
little light on the sum distribution problem. However, the extraordinary nonuniqueness of the lognormal moment problem is apparent.

\section{Evaluation of the characteristic function as a series}

The integral (41) is a relatively elegant formula. A more pedestrian infinite series, complicated but rapidly convergent, can be derived directly from (41) after a few preliminaries.

The function $1 / \Gamma(z+1)$ has been extensively studied by Ryzhik and earlier by Nielsen [13]. It is an entire function (analytic in the finite plane) and so if

$$
1 / \Gamma(z+1)=\sum_{k=0}^{\infty} d_{k} z^{k} \quad \text { and } \quad G_{\rho}=\max _{|z|=\rho} \mid\left(\left.\Gamma(z+1)\right|^{-1}\right),
$$

then Cauchy's inequality states that $\left|d_{k}\right| \leq G_{\rho} / \rho^{k}$ for each $k$, and each $\rho>0$. This result is not sharp enough for our needs, and is improved below. According to Ryzhik [7] the coefficients $d_{k}$ are defined recursively by

$$
d_{0}=1,(n+1) d_{n+1}=\gamma d_{n}+\sum_{k=1}^{n}(-1)^{k} \zeta(k+1) d_{n-k} \text { for } n \geq 0,
$$

where $\gamma$ is Euler's constant and $\zeta(r)=\sum_{n=1}^{\infty} n^{-r}$ is the zeta function. Nielsen's recursion has been used by Fransén [6] to calculate $d_{1}, d_{2}, \ldots, d_{50}$ to 80 decimal places. The calculation was checked by using the functional identity

$$
\frac{1}{\Gamma(1+z) \Gamma(1-z)}=\sum_{k} d_{k} z^{k} \sum_{l} d_{l}(-z)^{l}=\frac{\sin \pi z}{\pi z},
$$

and the resulting numerical identities

$$
\sum_{l=0}^{2 m}(-1)^{l} d_{l} d_{2 m-l}=\frac{(-1)^{m} \pi^{2 m}}{(2 m+1) !}, \quad m=1,2,3, \ldots
$$

High accuracy in $d_{1}, d_{2}, \ldots$ is necessary because they enter alternating sums in which they multiply factors which are quite large for some values of the parameters. Fortunately, the $d_{n}$ tend to zero about as fast as $1 / n !$.

The proof, suggested in part by Dr. Ryavec of USCB, depends on the fact that if $h(z)$ is any entire function with $h(z)=\sum_{n=0}^{\infty} e_{n} z^{n}$, then for $R>0$,

$$
\frac{1}{2 \pi} \int_{0}^{2 \pi}\left|h\left(R e^{i \theta}\right)\right|^{2} d \theta=\sum_{n=0}^{\infty}\left|e_{n}\right|^{2} R^{2 n}
$$


Hence in particular

but

$$
\left|d_{m}\right|^{2} R^{2 m} \leq \frac{1}{2 \pi} \int_{0}^{2 \pi}\left|\Gamma\left(1+r e^{i \theta}\right)\right|^{-2} d \theta
$$

$$
\begin{aligned}
\left|\Gamma\left(1+r e^{i \theta}\right)\right|^{-1} & \leq|\Gamma(1-R)|^{-1} \\
& =|\Gamma(R)|\left|\frac{\sin \pi R}{\pi R}\right| \leq \frac{\Gamma(R)}{\pi} \quad \text { for } R>1,
\end{aligned}
$$

as a rough estimate, so that $\left|d_{m}\right|^{2} R^{2 m} \leq\{\Gamma(R) / \pi\}^{2}$, and thus for each $R>1$,

$$
\begin{aligned}
\pi\left|d_{m}\right| \leq \Gamma(R) / R^{m} & =\leq(R / e)^{R} \sqrt{2 \pi / R} / R^{m} \\
& =\sqrt{2 \pi} R^{R-m-1 / 2} e^{-R} \\
& =\sqrt{2 \pi} \exp \left(B_{m}(R)\right) \quad \text { for all } R>1,
\end{aligned}
$$

where $B_{m}(R)=(R-m-1 / 2) \log R-R$.

We now choose $R(m)>1$ to minimise the right side of the inequality (53) for fixed $m$. Differentiation of $B_{m}(r)$ yields $B_{m}^{\prime}(R)=\log R-(m+1 / 2) / R$, and so $B_{m}^{\prime \prime}(R)=(1 / R)+(m+1 / 2) / R^{2}>0$.

The solution of the equation $R(m) \log R(m)=m+1 / 2$ would therefore provide a minimum to $B_{m}(R)$. This transcendental equation is difficult to solve, but suggests the cruder choice $R_{0}(m)=m / \log m$. Now, for each $\varepsilon>0$,

$$
\begin{aligned}
B_{m}\left(R_{0}(m)\right)= & \left(\frac{m}{\log m}-m-1 / 2\right)(\log m-\log \log m)-\frac{m}{\log m} \\
= & -m \log m+m-\frac{m}{\log m}(1+\log \log m) \\
& -1 / 2 \log m+(m+1 / 2) \log \log m \\
\leq & -(1-\varepsilon)(m \log m-m+1 / 2 \log m)
\end{aligned}
$$

for $m$ sufficiently large, since $m / \log m, m \log \log m$, and $m \log \log m / \log m$ are dominated asymptotically by $m, m \log m$, and $m \log m$ respectively. From (53),

$$
\begin{aligned}
\left|d_{m}\right| & \leq \sqrt{2 \pi} \exp \{-(1-\varepsilon)(m \log m-m+1 / 2 \log m)\} \\
& =\sqrt{2 / \pi}\left\{\left(\frac{e}{m}\right)^{m} \frac{1}{\sqrt{m}}\right\}^{1-\varepsilon} \\
& =O\left\{\left(\frac{m}{e}\right)^{m} \sqrt{2 \pi m}\right\}^{-(1-\varepsilon)} .
\end{aligned}
$$

Thus $\left|d_{m}\right|=O\left((m !)^{-(1-\varepsilon)}\right)$ for each $\varepsilon$, which justifies the earlier remark that $\left|d_{m}\right|$ decreases about as fast as $1 / m !$. A more precise bound is obtainable but unnecessary, in view of the application. 
A more elegant proof is based on a formula of Levin [11] in his book on meromorphic functions, namely $\rho=\varlimsup_{n \rightarrow \infty}\left(n \log (1 / n) / \log \left|d_{n}\right|\right)$, where $\rho$ is the "type" of $\psi(z)=1 / \Gamma(1+z)$. Since $\psi(z)$ has Weierstrass factors of order 1 , and its set of zeros is of order 1 , its type $\rho=1$. Hence for each $\varepsilon>0$, there is an $n(\varepsilon)$ such that $n \log (1 / n) \leq(1+\varepsilon) \log \left|d_{n}\right|$ for all $n>n(\varepsilon)$, which means

$$
\left|d_{n}\right| \leq n^{-n(1+\varepsilon)}
$$

and so $\psi(z)$ is convergent for all $z$.

Moreover, if $\psi_{m}(z)=\sum_{j=0}^{m} d_{j} z^{j}$ is a truncated expansion of $\psi(z)$, then

(a) $\left|\psi_{m}(z)-\psi(z)\right| \rightarrow 0$ inside any compact set uniformly as $m \rightarrow \infty$,

(b) $\psi(z)$ and $\psi(z)-\psi_{m}(z)$ are entire functions of type 1, and finally

(c) $\left|\psi(z)-\psi_{m}(z)\right| \leq C^{\prime} \exp \left(A^{\prime}|z|\right)$ for some positive constants $C^{\prime}, A^{\prime}$ for large $|z|$.

Clearly

$$
1 / \Gamma(1-z)=\sum_{l=0}^{\infty}(-1)^{l} d_{l} z^{\prime}
$$

Thus formally, on insertion of (55) in (40) and interchanging the order of integration and summation,

$$
\phi\left(0, \sigma^{2}, t\right)=1 / 2 \sum_{l=0}^{\infty}(-1)^{l} d_{l} \int_{k-i \infty}^{k+i \infty} z^{l} \exp \left\{\sigma^{2} z^{2} / 2-z(\log t+\pi i / 2)\right\} d z
$$

The interchange of order in formulas (31) and (56) can now be justified, since the exact expressions involved are finally available. To prove (31), consider from (32) and (38) and (41) the equivalent problem of interchange in

$$
\begin{aligned}
\int_{k-i \infty}^{k+i \infty} & \sum_{m=0}^{\infty} d_{m}(-1)^{m} z^{m} \exp \left\{\sigma^{2} z^{2} / 2-z\left(\log t+\frac{\pi i}{2}\right)\right\} d z \\
= & \sum_{m=0}^{\infty} d_{m}(-1)^{m} \int_{k-i \infty}^{k+i \infty} z^{m} \exp \left\{\sigma^{2} z^{2} / 2-z\left(\log t+\frac{\pi i}{2}\right)\right\} d z
\end{aligned}
$$

By the Lebesgue domination theorem, if

$$
\psi(-z)=\sum_{m} d_{m}(-1)^{m} z^{m}, \quad \psi_{n}(-z)=\sum_{m=0}^{n} d_{m}(-1)^{m} z^{m}
$$

and if

$$
\begin{aligned}
& \left|\psi_{n}(-z)\right|\left|\exp \left(\sigma^{2} z^{2} / 2-z\left(\log t+\frac{\pi i}{2}\right)\right)\right| \\
& \quad=\left|\psi_{n}(-k-i u)\right| \exp \left(\sigma^{2}\left(k^{2}-u^{2}\right) / 2-k \log t+\pi u / 2\right) \\
& \quad \leq q_{t}(u) \quad \text { for all } n,
\end{aligned}
$$


where $z=k+i u, u$ is real, and $\int_{-\infty}^{\infty} q_{t}(u) d u<\infty$, then

$$
\begin{aligned}
\lim _{n \rightarrow \infty} & \int_{k-i \infty}^{k+i \infty} \psi_{n}(-z) \exp \left(\sigma^{2} z^{2} / 2-z\left(\log t+\frac{\pi i}{2}\right)\right) d z \\
& =\sum_{l=0}^{\infty}(-1)^{l} d_{l} \int_{k-i \infty}^{k+i \infty} z^{l} \exp \left(\sigma^{2} z^{2} / 2-z\left(\log t+\frac{\pi i}{2}\right)\right) d z .
\end{aligned}
$$

Now by (54) et seq, take

$$
\begin{aligned}
q_{t}(u)= & \left(|\psi(-k-i u)|+\sup _{n \geq n_{0}}\left|\psi_{n}(-k-i u)-\psi(-k-i u)\right|\right) \\
& \times \exp \left\{\sigma^{2}\left(k^{2}-u^{2}\right) / 2-k \log t+\pi u / 2\right\}
\end{aligned}
$$

Clearly $q_{t}(u)$ is bounded on compact sets, and also $q_{t}(u)$ is bounded by

$$
C^{\prime \prime} \exp \left(A^{\prime \prime}\left(k^{2}+u^{2}\right)^{1 / 2}+\sigma^{2}\left(k^{2}-u^{2}\right) / 2-k \log t+\pi u / 2\right)
$$

for large $u$. The last exponential is bounded by $D \exp \left(-\sigma^{2} u^{2} / 4\right)$ for large $u$, as can be seen by comparing $\pi u / 2+A^{\prime \prime}\left(k^{2}+u^{2}\right)^{1 / 2}$ with $\sigma^{2} u^{2} / 4$ for large $u$. Hence $\int_{-\infty}^{\infty} q_{l}(u) d u<\infty$.

From (56), we find, using the same technique as in deriving (36), which depends here on the fact that $N e^{l-a N^{2}} \rightarrow 0$, for each $l$ as $N \rightarrow \infty$,

$$
\begin{aligned}
\int_{k-1 \infty}^{k+i \infty} z^{l} \exp \left(a z^{2}+\xi z\right) d z & =i(\pi / a)^{1 / 2}\left(\frac{\partial}{\partial \xi}\right)^{l} \exp \left(-\xi^{2} / 4 a\right) \\
& =i(\pi / a)^{1 / 2}(4 a)^{-l / 2}(-1)^{l} H_{l}(\xi / \sqrt{4 a}) \cdot \exp \left(-\xi^{2} / 4 a\right),
\end{aligned}
$$

using Rodrigues' formula for Hermite polynomials. Thus for $t>0$,

$$
\begin{aligned}
\phi\left(0, \sigma^{2}, t\right)= & \left\{\pi / 2 \sigma^{2}\right\}^{1 / 2} \exp \left\{-(\log t+\pi i / 2)^{2} / 2 \sigma^{2}\right\} \\
& \cdot \sum_{l=0}^{\infty}\left(-1^{\prime} d_{1}\left(2 \sigma^{2}\right)^{-l / 2} H_{l}\left\{(\log t+\pi i / 2) / \sigma \cdot 2^{1 / 2}\right\}\right.
\end{aligned}
$$

Both from a theoretical and a practical (computing) viewpoint, the rapidity of convergence of the series (58) is rather important. We have in (54) an estimate of $\left|d_{m}\right|$. It is now necessary to estimate $H_{m}\left\{(\log t+\pi i / 2) / \sigma \cdot 2^{1 / 2}\right\}$. Fortunately, an excellent estimate is available from the paper of Sansone [15], for Hermite polynomials of complex arguments and large order. This has the form

$\left|f_{m}(u+i v)\right| \leq 2 N^{-1 / 4} \exp \left\{|v|\left(N^{1 / 2}+\left(u^{2}+v^{2}\right) N^{-1 / 2}\right)\right\}\left(\pi^{-1 / 2} 2^{3 / 4}+N^{-1 / 4}|u|^{5 / 2}\right)$

where $N=2 m+1$, and $f_{m}(z)=\pi^{-1 / 4}\left(2^{m} m !\right)^{-1 / 2} \exp \left(-z^{2} / 2\right) H_{m}(z)$. 
It follows that, for $u$ and $v$ independent of $m$,

$$
\left|H_{m}(u+i v)\right|=O\left(2^{m / 2}(m !)^{1 / 2} m^{-1 / 4} \exp \left[(2 m+1)^{1 / 2}|v|\right]\right.
$$

and so

$$
\begin{aligned}
D_{m}(\sigma) & =\left|d_{m}\right|\left|2 \sigma^{2}\right|^{-m / 2}\left|H_{m}(\log t+\pi i / 2) / \sigma^{1 / 2}\right| \\
& =O\left\{\left(e^{\varepsilon+1 / 2}\right)^{m} \cdot \sigma^{-m} \cdot m^{\varepsilon m+1 / 2(\varepsilon-m-1)} \cdot \exp \left(\pi(w m+1) / \sigma\left(8 \sigma^{2}\right)^{1 / 2}\right\},\right.
\end{aligned}
$$

which is roughly dominated by $m^{-m / 2}$. More precisely, for $\sigma \geq 1, D_{m}(\sigma)$ is trivially $O\left(m^{-m / 2+2 e m}\right)$. For $\sigma<1$, there is an $m_{0}(\sigma, \varepsilon)$ and a $k(\sigma)$ such that $D_{m}(\sigma)$ is bounded by $k(\sigma) m^{-m / 2+2 e m}$ for $m>m_{0}(\sigma, \varepsilon)$, so that the same estimate holds, but not uniformly in $\sigma$. The terms in (58) thus decrease almost as fast as $(m !)^{-1 / 2}$, for large $m$.

In practice, 50 terms of the series (58) individually computed to $80 \mathrm{dec}-$ imal places to compensate for the oscillatory nature of the terms (by Dr. J. Wayman) suffices to give four place accuracy for $\sigma>0.1$, but the number of terms required rises quite rapidly as $\sigma \rightarrow 0$. The series for $\phi\left(0, \sigma^{2}, t\right)$ is uniformly and absolutely convergent for $t$ in any compact subset of $(0, \infty)$ and defines a continuous function of $t$ on $(0, \infty)$, and by the conjugate symmetry principle, on $(-\infty, 0)$. Since $\left|e^{i x t} p(x)\right| \leq p(x)$ and $\int_{0}^{\infty} p(x) d x=1$ and

it follows that

$$
\int_{0}^{\infty} \lim _{t \rightarrow 0^{+}}\left(e^{i x t} p(x)\right) d x=\int_{0}^{\infty} p(x) d x=1,
$$

$$
\lim _{t \rightarrow 0^{+}} \phi\left(0, \sigma^{2}, t\right)=1=\lim _{t \rightarrow 0^{-}} \phi\left(0, \sigma^{2}, t\right)
$$

and so $\phi$ is continuous at $t=0$ and hence for all $t$.

The series (58) combines $\log t$ and $\pi i / 2$ intimately. Their effects can be separated by the addition formula

$$
H_{l}\left(\frac{u+v}{\sqrt{2}}\right)=2^{-l / 2} \sum_{m=0}^{l}\left(\begin{array}{c}
l \\
m
\end{array}\right) H_{m}(u) H_{l-m}(v)
$$

which yields

$$
H_{l}\left(\frac{\log t+\pi i / 2}{\sqrt{2} \sigma}\right)=2^{-l / 2} \sum_{m=0}^{\infty} H_{m}\left(\frac{\log t}{\sigma}\right) H_{l-m}\left(\frac{\pi i}{2 \sigma}\right) .
$$

Returning to the case $\mu \neq 0$, we have

$$
\begin{aligned}
\phi\left(\mu, \sigma^{2}, t\right)= & \left(\frac{\pi}{2 \sigma^{2}}\right)^{1 / 2} \exp \left(-\frac{(\log t+\mu+\pi i / 2)^{2}}{2 \sigma^{2}}\right) \\
& \times \sum_{l=0}^{\infty}(-1)^{l} d_{l}\left(2 \sigma^{2}\right)^{-l / 2} H_{l}\left(\frac{\log t+\mu+\pi i / 2}{\sigma \sqrt{2}}\right) .
\end{aligned}
$$


By two applications of (61), the effects of $\mu, \log t$, and $\pi i / 2$ on the series (63) can be separated, at the cost of inserting a finite double sum into the infinite series.

The leading term in (63) is

$$
\phi_{0}(t)=\left(\pi /\left(2 \sigma^{2}\right)\right)^{1 / 2} \exp \left(-(\log t+\mu+\pi i / 2)^{2} /\left(2 \sigma^{2}\right)\right) .
$$

The inverse transform of $\phi_{0}(t)$ is similar to the integral for $\phi(t)$ itself, but slightly worse because of the complex power and so does not define a nice approximation of $p\left(\mu, \sigma^{2}, x\right)$.

The results for the sum of just two lognormal variables are series considerably more complicated than (60), but of the same general character. In the next section, the functional differential equation for $\phi$ is exploited to yield a functional equation for the embedding function, a natural enough generalisation of the sum distribution. This in turn permits a general expression for the sum distribution as a mixture, both discrete and continuous, of lognormal distributions with the same " $\sigma^{2}$ " parameter but different, complex-valued, " $m$ " parameters, as in fact suggested long ago by $\mathrm{H}$. Cramér. The explicit weights, etc. are determined in the sequel II in terms of other classical functions, Stirling numbers, and a new set of polynomials.

\section{Application of functional differential equations to the embedding and sum distributions}

If $X_{1}, X_{2}$ are independent lognormal variables with densities $p_{1}, p_{2}$, parameters $\mu_{1}, \sigma_{1}^{2}$ and $\mu_{2}, \sigma_{2}^{2}$, then the density $h_{2}$ of $y_{2}=X_{1}+X_{2}$ is given by the convolution integral $h_{2}(y)=\int_{0}^{y} p_{1}(y-x) p_{2}(x) d x$. We wish to obtain some information on $h_{2}$ without recourse to tedious analytical methods. (This is found in the sequel II.) One approach is through a generalisation of $h_{2}$ which lends itself to use of the functional differential equation (16), exploited successfully in Sections 2 and 3.

We define the 2-embedding function $g_{2}\left(x, \theta_{1}, \theta_{2}\right)$ by the integral

$$
g_{2}\left(x, \theta_{1}, \theta_{2}\right)=(2 \pi)^{-1} \int_{-\infty}^{\infty} \phi_{1}\left(\theta_{1} t\right) \phi_{2}\left(\theta_{2} t\right) \exp (-i x t) d t
$$

where $\phi_{j}(t)=\phi\left(\mu_{j}, \sigma_{j}^{2}, t\right)$, and $\theta_{1}, \theta_{2}$ are real.

Clearly, by substitution in (64),

$$
g_{2}(x, 1,1)=(2 \pi)^{-1} \int_{-\infty}^{\infty} \phi_{1}(t) \phi_{2}(t) \exp (-i x t) d t=h_{2}(x)
$$


Also

$$
\begin{array}{rlrl}
g_{2}\left(x, \theta_{1}, 0\right) & =(2 \pi)^{-1} \int \phi_{1}\left(\theta_{1} t\right) \exp (-i x t) d t \\
& =\theta_{1}^{-1} p_{1}\left(x \theta_{1}^{-1}\right), & & \theta_{1} \neq 0, \\
g_{2}\left(x, 0, \theta_{2}\right) & =\theta_{2}^{-1} p_{2}\left(x \theta_{2}^{-1}\right), & & \theta_{2} \neq 0 .
\end{array}
$$

Thus $g_{2}$ behaves like $h_{2}$, but has some useful extra parameters to operate with. By parts integration,

$$
\begin{aligned}
& g_{2}\left(x, \theta_{1}, \theta_{2}\right)= \\
& -\frac{1}{2 \pi x} \lim _{A \rightarrow \infty}\left[\left\{\exp (-i x t) \phi_{1}\left(\theta_{1} t\right) \phi_{2}\left(\theta_{2} t\right)\right\}_{-A}^{A}\right. \\
& \left.\quad-\frac{1}{2 \pi} \int_{-A}^{A}(-i x)^{-1} \exp (-i x t) d\left(\phi_{1}\left(\theta_{1} t\right) \phi_{2}\left(\theta_{2} t\right)\right)\right] .
\end{aligned}
$$

By (11), (12), the first limit is zero if $\theta_{1} \neq 0$ or $\theta_{2} \neq 0$, and the second limit yields

$$
\begin{aligned}
g_{2}\left(x, \theta_{1}, \theta_{2}\right)= & (2 \pi i x)^{-1} \\
& \times \int_{-\infty}^{\infty} \exp (-i x t)\left\{\theta_{1} \phi_{1}^{\prime}\left(\theta_{1} t\right) \phi_{2}\left(\theta_{2} t\right)+\theta_{2} \phi_{2}^{\prime}\left(\theta_{2} t\right) \phi_{1}\left(\theta_{1} t\right)\right\} d t .
\end{aligned}
$$

The functional differential equation (16) immediately leads to the equation

$$
\begin{aligned}
x g_{2}\left(x, \theta_{1}, \theta_{2}\right) & =(2 \pi)^{-1} \int_{-\infty}^{\infty} \exp (-i x t)\left[\theta_{1} K_{1} \phi_{1}\left(L_{1} t\right)+\theta_{2} K_{2} \phi_{2}\left(L_{2} t\right)\right] d t \\
& =\theta_{1} K_{1} g_{2}\left(x, L_{1} \theta_{1}, \theta_{2}\right)+\theta_{2} K_{2} g_{2}\left(x, \theta_{1}, L_{2} \theta_{2}\right)
\end{aligned}
$$

where

$$
K_{j}=\exp \left(\mu_{j}+\sigma_{j}^{2} / 2\right) \quad \text { and } \quad L_{j}=\exp \left(\sigma_{j}^{2}\right) .
$$

The functional equation (69) can be fairly completely solved. First we write

$$
r_{j}(x)=x p_{j}(x) \text {. }
$$

From the partial boundary condition

$$
\begin{aligned}
x \theta_{1}^{-1} p_{1}\left(x \theta_{1}^{-1}\right) & =x g_{2}\left(x, \theta_{1}, 0\right) \\
& =\theta_{1} K_{1} g_{2}\left(x, L_{1} \theta_{1}, 0\right) \\
& =K_{1} L_{1}^{-1} p_{1}\left(x L_{1}^{-1} \theta_{1}^{-1}\right)
\end{aligned}
$$

we infer

$$
\left\{\begin{array}{l}
x r_{1}\left(x \theta_{1}^{-1}\right)=K_{1} \theta_{1} r_{1}\left(x L_{1}^{-1} \theta_{1}^{-1}\right) \\
x r_{2}\left(x \theta_{2}^{-1}\right)=K_{2} \theta_{2} r_{2}\left(x L_{2}^{-1} \theta_{2}^{-1}\right) .
\end{array}\right. \text { and likewise }
$$


Now define a reduced embedding function $G_{2}\left(x, \theta_{1}, \theta_{2}\right)$ by

$$
g_{2}\left(x, \theta_{1}, \theta_{2}\right)=r_{1}\left(x \theta_{1}^{-1}\right) r_{2}\left(x \theta_{2}^{-1}\right) G_{2}\left(x, \theta_{1}, \theta_{2}\right)
$$

and conclude from (73), (69) that

$$
G_{2}\left(x, \theta_{1}, \theta_{2}\right)=G_{2}\left(x, L_{1} \theta_{1}, \theta_{2}\right)+G_{2}\left(x, \theta_{1}, L_{2} \theta_{2}\right),
$$

a somewhat more desirable functional equation than (69). We now try a solution of the form

$$
G_{2}\left(x, \theta_{1}, \theta_{2}\right)=\Re \sum_{m \in U(x)} \sum_{n \in V(x)} \int \psi_{m n}(x, d \alpha) \theta_{1}^{\beta_{m}(x, \alpha)} \theta_{2}^{y_{n}(x, \alpha)}
$$

where $\left\{\psi_{m n}(d \alpha)\right\}$ is a double sequence of complex-valued measures on $\Omega_{2}$ and $U(x), V(x)$ are sets of integers for each $x$. The dimensionality of $\alpha$ is as yet unspecified. The function sequences $\left\{\beta_{m}(x, \alpha)\right\},\left\{\gamma_{n}(x, \alpha)\right\}$ are complex-valued.

Clearly, we have

$$
\begin{aligned}
G_{2}\left(x, \theta_{1}, \theta_{2}\right)-G_{2}\left(x, L_{1} \theta_{1}, \theta_{2}\right)-G_{2}\left(x, \theta_{1}, L_{2} \theta_{2}\right) \\
\quad=\Re \int \sum_{m \in U(x)} \sum_{n \in V(x)} \psi_{m n}(x, d \alpha) \theta_{1}^{\beta_{m}(x, \alpha)} \theta_{2}^{\gamma_{n}(x, \alpha)}\left(1-L_{1}^{\beta_{m}(x, \alpha)}-L_{2}^{\gamma_{n}(x, a)}\right) .
\end{aligned}
$$

Thus (72) is satisfied whenever

$$
L_{1}^{\beta_{m}(x, \alpha)}+L_{2}^{\gamma_{n}(x, \alpha)}=1 \quad \text { for each } m, n, \alpha, x .
$$

A general solution of $(75)$ is

$$
\left\{\begin{array}{l}
\beta_{m}(x, \alpha)=\{\log \varepsilon(x, \alpha)+2 \pi i m\} / \log L_{1} \\
\gamma_{n}(x, \alpha)=\{\log (1-\varepsilon(x, \alpha))+2 \pi i n\} / \log L_{2}
\end{array}\right.
$$

where $0<\varepsilon(x, \alpha)<1$ for each $x, \alpha$, and $m, n$ are integers.

We note from the definition of $g_{2}$ that for $c>0$

$$
g_{2}\left(c x, d_{1} \theta_{1}, d_{2} \theta_{2}\right)=c^{-1} g_{2}\left(x, d_{1} \theta_{1} / c, d_{2} \theta_{2} / c\right)
$$

and thus also

$$
G_{2}\left(c x, d_{1} \theta_{1}, d_{2} \theta_{2}\right)=c^{-1} G_{2}\left(x, d_{1} \theta_{1} / c, d_{2} \theta_{2} / c\right) .
$$

From (77), we have

$$
\begin{aligned}
\Re \int & \sum_{m \in U(c x)} \sum_{n \in V(c x)} \psi_{m n}(c x, d \alpha)\left(d_{1} \theta_{1}\right)^{\beta_{m}(c x, \alpha)}\left(d_{2} \theta_{2}\right)^{\gamma_{n}(c x, \alpha)} \\
= & c^{-1} \mathfrak{R} \int \sum_{m \in U(x)} \sum_{n \in V(x)} \psi_{m n}(x, d \alpha)\left(d_{1} \theta_{1} / c\right)^{\beta_{m}(x, \alpha)} \cdot\left(d_{2} \theta_{2} / c\right)^{y_{n}(x, \alpha)}
\end{aligned}
$$


for all real $c, x$. It follows that

$$
\begin{aligned}
U(c x) & =U(x)=U, \quad V(c x)=V(x)=V, \\
\beta_{m}(c x, \alpha) & =\beta_{m}(x, \alpha)=\beta_{m}(\alpha), \quad \gamma_{n}(c x, \alpha)=\gamma_{n}(x, \alpha)=\gamma_{n}(\alpha), \\
\varepsilon(x, \alpha) & =\varepsilon(\alpha),
\end{aligned}
$$

are independent of the value of the first argument. Also

$$
\psi_{m n}(c x, d \alpha)=c^{-\delta_{m n}(\alpha)} \psi_{m n}(x, d \alpha)
$$

where

$$
\delta_{m n}(\alpha)=1+\beta_{m}(\alpha)+\gamma_{n}(\alpha) .
$$

Interchange of $c$ and $x$ in (82) yields the conclusion

$$
\psi_{m n}(x, d \alpha)=x^{-\delta_{m n}(\alpha)} \psi_{m n}(d \alpha)
$$

so that we have

$$
\begin{aligned}
g_{2}(x)= & x p_{1}(x) p_{2}(x) \Re \int_{\Omega_{2}} \sum_{m \in U} \sum_{n \in V} \psi_{m n}(d \alpha) \\
& \times\left(x^{-\{\log \varepsilon(\alpha)+2 \pi \imath m\} / \sigma_{1}^{2}}\right)\left(x^{-\{\log (1-\varepsilon(\alpha))+2 \pi i n\} / \sigma_{2}^{2}}\right) .
\end{aligned}
$$

This formula already shows that the density $g_{2}(x)$ is a continuous and double discrete mixture of lognormal densities each of which has the same $\sigma^{2}$ parameter, namely $\sigma_{1}^{2} \sigma_{2}^{2} /\left(\sigma_{1}^{2}+\sigma_{2}^{2}\right)$.

The same method, applied to a sum $Y_{k}=X_{1}+X_{2}+\cdots+X_{k}$ of independent lognormals of parameters $\left(\mu_{1}, \sigma_{1}^{2}\right),\left(\mu_{2}, \sigma_{2}^{2}\right), \ldots,\left(\mu_{k}, \sigma_{k}^{2}\right)$, yields for the sum density $g_{k}(x)$ the form

$$
\begin{aligned}
g_{k}(x)= & x^{k-1} p_{1}(x) p_{2}(x) \ldots p_{k}(x) \Re \int_{\Omega_{k}} \sum_{m_{1} \in U_{1}} \cdots \sum_{m_{k} \in U_{k}} \psi_{m_{1}, \ldots, m_{k}}(d \alpha) \\
& \times\left(x^{-\left(\log \varepsilon_{1}(\alpha) / \sigma_{1}^{2}+\cdots+\log \varepsilon_{k}(\alpha) / \sigma_{k}^{2}\right)}\right)\left(x^{-2 \pi i\left(m_{1} / \sigma_{1}^{2}+\cdots+m_{k} / \sigma_{k}^{2}\right)}\right)
\end{aligned}
$$

where $\varepsilon_{1}(\alpha)+\cdots+\varepsilon_{k}(\alpha)=1$, and $\Omega_{k}$ is a set. Thus $g_{k}$ is also a continuous and $k$-tuply discrete mixture of lognormal densities, each of which has the same " $\sigma^{2}$ parameter", $\left(\sum_{j=1}^{k} \sigma_{j}^{-2}\right)^{-1}$. The explicit determination of $\varepsilon(\alpha), U, V, \Omega_{2}$ and $\psi_{m n}(d \alpha)$ [or of $\varepsilon_{1}(\alpha), \ldots \varepsilon_{k}(\alpha), U_{1}, \ldots, U_{k}, \Omega_{k}$, $\psi_{m_{1}, \ldots, m_{k}}(d \alpha)$ for $k>2$ ] is more tedious and is left to II. The analytical results are not very illuminating, unfortunately, but are quite computable using multiple precision arithmetic.

Similar, though rather more complicated results have been obtained for "Einstein-normal" - or Johnson type 2 - random variables, in which the lognormal is modified by replacing the exponential (whose inverse is the 
logarithm) with a scaled hyperbolic tangent arising from "Einstein's addition rule"

$$
f\left(x_{1}+x_{2}\right)=\frac{f\left(x_{1}\right)+f\left(x_{2}\right)}{1+f\left(x_{1}\right) f\left(x_{2}\right) / c^{2}} .
$$

This arises in the study of "naturally" truncated normal variables.

The author wishes to thank Dr. Uppuluri of ORNL, and Professors Pesotchinsky, Rao, Ryavec, and Kotlarski of USCB, for careful reading of this paper, and for their encouragement in pursuing these rather elusive problems.

For those who wish to pick out the key formulas, (15) and (16) are the functional equations for the characteristic functions, (40) and (41) are their Laplace transform representations, (42) and (46) give the moment nonuniqueness example, (49) and (50) define the Nielsen coefficients which enter the Hermite expansion (58), and the estimates which establish convergence of (58), are in (53) et seq and (59) et seq. As for the partially determined sum distribution, (64), (69), (74), (75), (78), (79), (85), (86), are the high points leading to (87).

\section{References}

[1] F. H. Brownell, Pacific J. Math. 5 (1955) 484-491.

[2] N. J. de Bruijn, Nederl. Akad. Wetensch Proc. Series A 56 (1953) 449-458; Indagationes Math 15 (1953) 459-464.

[3] T. Carleman, Les fonctions quasi-analytiques (Gauthier-Villars, Paris, 1926).

[4] E. L. Crow and K. Shimizu, Lognormal distributions: Theory and applications (Dekker, New York, 1988).

[5] A. Erdelyi, et al, Tables of integral transforms (Vol. 1, McGraw-Hill, New York, 1954).

[6] A. Fransên and S. Wrigge, Math. of Comp. 34 (1980) 553-566.

[7] I. Gradshtein and I. Ryzhik, Tables of integrals, series and products (2nd transl. ed., Academic Press, New York, 1980).

[8] P. Holgate, "The lognormal characteristic function", Comm Stat. Theory (In Press).

[9] M. Krein, C. R. (Doklady) Acad. Sci. URSS (NS) 40 (1945) 306-309.

[10] R. B. Leipnik, “The lognormal distribution and strong nonuniqueness of the moment problem", J. Prob. Appl. (1981) 863-865.

[11] B. J. Levin, Distribution of zeros of entire functions, v5., Translations of Mathematical Monographs, AMS (1964).

[12] W. Magnus et al., Formulas and theorems for the special functions of mathematical physics (Springer, New York, 1966).

[13] N. Nielsen, Handbuch der Theorie der Gammafunktion (Teubner, Leipzig, 1906).

[14] E. Pinney, Ordinary difference-differential equations (University of California, Berkeley, 1958).

[15] G. Sansone, Orthogonal functions (Interscience, New York, 1959).

[16] E. G. Titchmarsh, The theory of fourier integrals (Oxford University Press, Oxford, 1948). 